To appear in the Proceedings of

International Symposium on Symbolic

and Algebraic Computation

Lille, France, July 2002

\title{
On the Efficiency and Optimality of Dixon-based Resultant Methods*
}

\author{
Arthur Chtcherba \\ Deepak Kapur
}

\author{
Department of Computer Science \\ University of New Mexico \\ Albuquerque, NM 87131 \\ e-mail: \{artas,kapur\}@cs.unm.edu
}

\begin{abstract}
Structural conditions on polynomial systems are developed for which the Dixon-based resultant methods often compute exact resultants. For cases when this cannot be done, the degree of the extraneous factor in the projection operator computed using the Dixon-based methods is typically minimal. A method for constructing a resultant matrix based on a combination of Sylvester-dialytic and Dixon methods is proposed. A heuristic for variable ordering for this construction often leading to exact resultants is developed.
\end{abstract}

Keywords: Resultant, Dixon Method, Bezoutians, Sylvester-type matrices, Extraneous Factor, Dialytic Method, Multiplier Matrices, BKK Bound, Support

\section{Introduction}

Resultant matrices based on the Dixon formulation have turned out to be quite efficient in practice for simultaneously eliminating many variables on a variety of examples from different application domains; for details and comparison with other resultant formulations and elimination methods, see [KS95, CK02a] and http://www.cs.unm.edu/ artas. Necessary conditions can be derived on parameters in a problem formulation under which the associated polynomial system has a solution. A main limitation of this approach is that often an extraneous factor is generated [KS97] with no relation with the resultant of a given polynomial system. This paper reports results about polynomial systems for which the Dixon formulation leads to the exact resultant (without any extraneous factor). These results generalize the earlier results of the authors for bivariate polynomial systems [CK02a] as well as the results of Chionh [Chi01] and Zhang and Goldman [ZG00] on corner-cut supports for bivariate polynomial systems. A method for determining a variable ordering is proposed such that the projection operator obtained using the Dixon formulation using the variable ordering often has the smallest degree. This method has the distinct advantage of generalizing most known cases of unmixed polynomial systems (such as $n$-degree systems as well as systems with corner-cut supports) for which the Dixon formulation is known to compute the resultant exactly [CK00a, CK02a].

In an earlier paper [CK02a], the bivariate case was completely analyzed by the authors. The notion of a support-interior point with respect to the support of a polynomial system was introduced, and it was shown that unlike other points in the Newton polytope (convex hull) of the support, including monomials corresponding to support-interior points do not change the degree of the extraneous factor. Thus, given a generic unmixed polynomial system with a given support, the projection operator computed using the

\footnotetext{
*This research is supported in part by NSF grant nos. CCR-9996144, CCR-0203051, CDA-9503064, and a grant from the Computer Science Research Institute at the Sandia National Labs.
} 
Dixon formulation has the same degree as that of a related generic unmixed polynomial system with a support which along with the original support, also includes all support-interior points. The definition of a support-interior point (with the same desired properties) is generalized to an arbitrary dimension.

Sylvester-type multiplier matrices based on the Dixon formulation are introduced using a general construction which turns out to be effective especially for mixed polynomial systems. This construction generalizes a construction discussed in our previous work [CK00b]. Multiplier sets for each polynomial in a given polynomial system are computed, generating a matrix whose determinant (or the determinant of a maximal minor) includes the resultant. It is shown that an arbitrary monomial can be used to do this construction. For the unmixed case, this construction is shown to be optimal if the monomial used is from the support of the polynomial system. To be precise, given a generic unmixed polynomial system, if the Dixon formulation produces a Dixon matrix whose determinant is the resultant, then the Sylvester-type multiplier matrices (henceforth, called the Dixon multiplier matrices) based on the proposed construction also have the resultant as their determinants. In case the Dixon matrix is such that the determinant of the maximal minor has an extraneous factor besides the resultant, the Dixon multiplier matrix does not have an extraneous factor of higher degree. Thus, no additional extraneous factor is contributed to the result by the proposed construction.

The main advantage of using the Dixon multiplier matrices over the associated Dixon matrices is (i) in the mixed case, the Dixon multiplier matrices can have resultants as their determinants, whereas the Dixon matrices often have determinants which includes along with the resultants, extraneous factors; further, if the determinant of a Dixon multiplier matrix has an extraneous factor with the resultant, the degree of the extraneous factor is lower than the degree of the extraneous factor appearing in the determinant of the Dixon matrix, (ii) the Dixon multiplier matrices can be stored and computed more efficiently, given that the entries are either zero or the coefficients of the monomials in the polynomials; this is in contrast to the entries of the Dixon matrices which are determinants in the coefficients. This paper is a summary of results; details including proofs of the results reported in this paper can be found in $[\mathrm{CK} 02 \mathrm{~b}]$ and $[\mathrm{CK} 02 \mathrm{c}])$.

\section{Multivariate Resultants}

Consider a system of polynomial equations $\mathcal{F}=\left\{f_{0}, \ldots, f_{d}\right\}$,

$$
f_{0}=\sum_{\alpha \in \mathcal{A}_{0}} c_{0, \alpha} \mathbf{x}^{\alpha}, \quad \cdots, \quad f_{d}=\sum_{\alpha \in \mathcal{A}_{d}} c_{d, \alpha} \mathbf{x}^{\alpha}
$$

where for each $i \in\{0, \ldots, d\}$, support $\mathcal{A}_{i} \subset \mathbb{N}^{d}$ and monomial $\mathbf{x}^{\alpha}=\left(x_{1}^{\alpha_{1}} x_{2}^{\alpha_{2}} \cdots x_{d}^{\alpha_{d}}\right)$ where $\mathbf{c}=\left(c_{i, \alpha}\right)$ are parameters. We will denote by $\mathcal{A}=\left\langle\mathcal{A}_{0}, \mathcal{A}_{1}, \ldots, \mathcal{A}_{d}\right\rangle$, the support of the polynomial system $\mathcal{F}$.

The goal is to derive condition on parameters $\mathbf{c}$ so that the polynomial system $\mathcal{F}=0$ has a solution. This problem can be viewed as the elimination of variables from the polynomial system. Such a condition exists for a large family of polynomial systems, and is called the resultant of the polynomial system. Since the number of equations is more than the number of variables, in general, for arbitrary values of $c_{i, \alpha}$, the polynomial system $\mathcal{F}$ does not have any solution. Therefore resultant is a necessary and sufficient condition to have a solution. Depending on the solution space (variety) one is interested in, different resultants can be computed. Here we will assume that solution space is projective closure of the algebraic torus $\left(\mathbb{C}^{*}\right)^{d}$. The reader can consult [EM99, GKZ94, Stu94, BEM00] for a detailed background.

\subsection{Degree of the Resultant}

The convex hull of the support of a polynomial $f$ is called its Newton polytope, and will be denoted as $\mathcal{N}(f)=\operatorname{Conv}(\mathcal{A})$, where $\mathcal{A}$ is the support of $f$. The Newton polytopes of a polynomial system determines the number of its roots in the toric variety. In a generic setting, it is possible to write a formula for the number of toric solutions of a polynomial system using the theory of mixed volumes of the associated Newton polytopes. 
Let $\mu\left(\mathcal{A}_{0}, \ldots, \mathcal{A}_{i-1}, \mathcal{A}_{i+1}, \ldots, \mathcal{A}_{d}\right)$ be the number of solutions of a generic polynomial system $\mathcal{F}-\left\{f_{i}\right\}$ in $\left(\mathbb{C}^{*}\right)^{d}$. This bound is called the BKK bound after Bernstein, Kouchnirenko and Khovansky who studied the number of solutions of a polynomial system in 70 's over the toric variety. In a generic unmixed case when $\mathcal{N}\left(f_{i}\right)=\mathcal{N}\left(f_{j}\right)$, for any $i \in\{0, \ldots, d\}$,

$$
\mu\left(\mathcal{A}_{0}, \ldots, \mathcal{A}_{i-1}, \mathcal{A}_{i+1}, \ldots, \mathcal{A}_{d}\right)=d ! \operatorname{Vol}\left(\mathcal{A}_{i}\right) .
$$

see [CLO98]. In the resultant, the degree of the coefficients of $f_{0}$ is equal to the number of the common roots of the rest of the polynomials. It is possible to choose any $f_{i}$ and the resultant expression can be expressed by substituting in $f_{i}$, the common roots of the remaining polynomial multiplied by a factor independent of $f_{i}$ [PS93]. The degree of the coefficients of $f_{i}$ in the resultant thus equals the number of roots of the remaining set of polynomials. We abuse the notation somewhat and denote the BKK bound of a $d+1$ polynomial system by $\left\langle b_{0}, b_{1}, \ldots, b_{d}\right\rangle$ as well as $B$, where $b_{i}=\mu\left(\mathcal{A}_{0}, \ldots, \mathcal{A}_{i-1}, \mathcal{A}_{i+1}, \ldots, \mathcal{A}_{d}\right)$ and $B=\sum_{i=0}^{d} b_{i}$.

\subsection{Resultant Matrices}

One way to compute the resultant of a given polynomial system is to construct a matrix with a property that whenever the polynomial system has a solution, such a matrix has a deficient rank, thereby implying that the determinant of any maximal minor is a multiple of the resultant. The BKK bound then serves as the lower bound on the size of such a matrix.

A simple way to construct a resultant matrix is to use the dialytic method, i.e., multiply each polynomial with a finite set of monomials, and rewrite the resulting system in the matrix form. We call such a matrix a multiplier matrix. This alone, however, does not guarantee that a matrix so constructed is a resultant matrix.

Definition 2.1 Given a set of polynomials $\left\{f_{1}, \ldots, f_{k}\right\}$ in variables $x_{1}, \ldots, x_{d}$ and finite monomials sets $X_{1}, \ldots, X_{k}$, where $X_{i}=\left\{\mathbf{x}^{\alpha} \mid \alpha \in \mathbb{N}^{d}\right\}$, denote by $X_{i} f_{i}=\left\{\mathbf{x}^{\alpha} f_{i} \mid \mathbf{x}^{\alpha} \in X_{i}\right\}$. The matrix representing the polynomial system $X_{i} f_{i}$ for all $i=1, \ldots, k$, can be written as

$$
\left(\begin{array}{c}
X_{1} f_{1} \\
X_{2} f_{2} \\
\vdots \\
X_{k} f_{k}
\end{array}\right)=M \times X=0
$$

where $X^{T}=\left(\mathbf{x}^{\beta_{1}}, \ldots, \mathbf{x}^{\beta_{l}}\right)$ such that $\mathbf{x}^{\beta} \in X$ if there exist $i$ such that $\mathbf{x}^{\beta}=\mathbf{x}^{\alpha} \mathbf{x}^{\gamma}$ where $\mathbf{x}^{\alpha} \in X_{i}$ and $\mathbf{x}^{\gamma} \in f_{i}$. Such matrices will be called as the multiplier matrices.

If a given multiplier matrix is square and non-singular, and the corresponding polynomial system has a solution which does not make the monomial vector $X$ identically zero, then its determinant is a multiple of the resultant over a variety that contains that solution. Furthermore, the requirement on the matrix to be non-singular (or even square) can be relaxed, as long as it can be shown that its rank becomes deficient whenever there exist a solution; in such cases, the multiple of the resultant can be extracted from a maximal minor of this matrix.

Note that such matrices are usually quite sparse: matrix entries are either zero or coefficients of the polynomials in the original system. Good examples of resultant multiplier matrices are Sylvester [Syl53] for the univariate case, and Macaulay [Mac16] as well Newton matrices of [CE00] for the multivariate case; they all differ only in the selection of multiplier sets $X_{i}$.

If the BKK bound of a given polynomial system is given by a tuple $\left\langle b_{0}, b_{1}, \ldots, b_{d}\right\rangle$, then $\left|X_{i}\right| \geq b_{i}$ and the matrix size must be at least $B$ (the sum of all the $b_{i}$ 's) for it to be a candidate for the resultant matrix of the polynomial system.

In the following sections, we show how the Dixon formulation can be used to construct multiplier matrices for the multivariate case. We first give a brief overview of the Dixon formulation, define the 
concepts of the Dixon polynomial and the Dixon matrix of a given polynomial system. Expressing the Dixon polynomial using the Cauchy-Binet expansion of determinants of a matrix turns out to be very useful for illustrating the dependence of the construction on the support of a given polynomial system.

\subsubsection{Dixon Matrix}

In [Dix08], Dixon generalized Bezout-Cayley's construction for computing the resultant of two univariate polynomials to the bivariate case. In [KSY94], Kapur, Saxena and Yang further generalized this construction to the general multivariate case; the concepts of Dixon polynomial and Dixon matrix were introduced as well. Below, the generalized multivariate Dixon formulation for simultaneously eliminating many variables from a polynomial system and computing its resultant is reviewed. More details can be found in [KS95].

In contrast to multiplier matrices, the Dixon matrix is dense since its entries are determinants of the coefficients of the polynomials in the original polynomial system. It has the advantage of being an order of magnitude smaller in comparison to a multiplier matrix, which is important as the computation of the determinant of a matrix with symbolic entries is sensitive to its size. The Dixon matrix is constructed through the computation of the Dixon polynomial, which is expressed in matrix form.

Let $\pi_{i}\left(\mathbf{x}^{\alpha}\right)=\bar{x}_{1}^{\alpha_{1}} \cdots \bar{x}_{i}^{\alpha_{i}} x_{i+1}^{\alpha_{i+1}} \cdots x_{d}^{\alpha_{d}}$, for $i \in\{0, \ldots, d\}$, and $\bar{x}_{i}$ 's are new variables; $\pi_{0}\left(\mathbf{x}^{\alpha}\right)=\mathbf{x}^{\alpha} . \pi_{i}$ is extended to polynomials in a natural way as: $\pi_{i}\left(f\left(x_{1}, \ldots, x_{d}\right)\right)=f\left(\bar{x}_{1}, \ldots, \bar{x}_{i}, x_{i+1}, \ldots, x_{d}\right)$.

Definition 2.2 Given a polynomial system $\mathcal{F}=\left\{f_{0}, \ldots, f_{d}\right\}$, where $\mathcal{F} \subset \mathbb{Q}[\mathbf{c}]\left[x_{1}, \ldots, x_{d}\right]$, define its Dixon polynomial as

$$
\theta\left(f_{0}, \ldots, f_{d}\right)=\prod_{i=1}^{d} \frac{1}{\bar{x}_{i}-x_{i}}\left|\begin{array}{cccc}
\pi_{0}\left(f_{0}\right) & \pi_{0}\left(f_{1}\right) & \cdots & \pi_{0}\left(f_{d}\right) \\
\pi_{1}\left(f_{0}\right) & \pi_{1}\left(f_{1}\right) & \cdots & \pi_{1}\left(f_{d}\right) \\
\vdots & \vdots & \ddots & \vdots \\
\pi_{d}\left(f_{0}\right) & \pi_{d}\left(f_{1}\right) & \cdots & \pi_{d}\left(f_{d}\right)
\end{array}\right| .
$$

Hence $\theta\left(f_{0}, f_{1}, \ldots, f_{d}\right) \in \mathbb{Q}[\mathbf{c}]\left[x_{1}, \ldots, x_{d}, \bar{x}_{1}, \ldots, \bar{x}_{d}\right]$, where $\bar{x}_{1}, \bar{x}_{2}, \ldots, \bar{x}_{d}$ are new variables.

The order in which original variables in $\mathbf{x}$ are replaced by new variables in $\overline{\mathbf{x}}$ is significant in the sense that Dixon polynomials computed using two different orderings may be different.

Definition 2.3 A Dixon polynomial $\theta\left(f_{0}, \ldots, f_{d}\right)$ can be written in bilinear form as

$$
\theta\left(f_{0}, f_{1}, \ldots, f_{d}\right)=\bar{X} \Theta X^{T},
$$

where $\bar{X}=\left[\overline{\mathbf{x}}^{\beta_{1}}, \ldots, \overline{\mathbf{x}}^{\beta_{k}}\right]$ and $X=\left[\mathbf{x}^{\alpha_{1}}, \ldots, \mathbf{x}^{\alpha_{l}}\right]$ are row vectors. The $k \times l$ matrix $\Theta$ is called the Dixon matrix.

Each entry in the matrix $\Theta$ is a polynomial in the coefficients of the original polynomials in $\mathcal{F}$; moreover its degree in the coefficients of any given polynomial is at most 1 . Therefore, the projection operator computed using the Dixon formulation can be of at most of degree $|X|$ in the coefficients of any single polynomial.

Below, we show a relationship between the support of a given polynomial system $\mathcal{A}=\left\langle\mathcal{A}_{0}, \ldots, \mathcal{A}_{d}\right\rangle$ and the support of its Dixon polynomial.

\subsubsection{Dixon Matrix \& Support of Polynomial System}

Proposition 2.1 [CK00a] Let $\mathcal{F}=\left\{f_{0}, f_{1}, \ldots, f_{d}\right\}$ be a polynomial system and let $\mathcal{A}=\left\langle\mathcal{A}_{0}, \ldots, \mathcal{A}_{d}\right\rangle$ be the support of $\mathcal{F}$. Let $\sigma=\left\langle\sigma_{0}, \ldots, \sigma_{d}\right\rangle \in \mathcal{A}$ be a support where $\sigma_{i} \in \mathcal{A}_{i}$ for $i=0, \ldots, d$. Then

$$
\theta\left(f_{0}, f_{1}, \ldots, f_{d}\right)=\sum_{\sigma \in \mathcal{A}} \sigma(\mathbf{c}) \sigma(\mathbf{x})
$$


where $\theta_{\sigma}=\sigma(\mathbf{c}) \sigma(\mathbf{x})$ and

$$
\sigma(\mathbf{c})=\left|\begin{array}{cccc}
c_{0, \sigma_{0}} & c_{0, \sigma_{1}} & \cdots & c_{0, \sigma_{d}} \\
c_{1, \sigma_{0}} & c_{1, \sigma_{1}} & \cdots & c_{1, \sigma_{d}} \\
\vdots & \vdots & \ddots & \vdots \\
c_{d, \sigma_{0}} & c_{d, \sigma_{1}} & \cdots & c_{d, \sigma_{d}}
\end{array}\right| \quad \text { and } \quad \sigma(\mathbf{x})=\prod_{i=1}^{d} \frac{1}{\bar{x}_{i}-x_{i}}\left|\begin{array}{cccc}
\pi_{0}\left(\mathbf{x}^{\alpha_{\sigma_{0}}}\right) & \pi_{0}\left(\mathbf{x}^{\alpha_{\sigma_{1}}}\right) & \cdots & \pi_{0}\left(\mathbf{x}^{\alpha_{\sigma_{d}}}\right) \\
\pi_{1}\left(\mathbf{x}^{\alpha_{\sigma_{0}}}\right) & \pi_{1}\left(\mathbf{x}^{\alpha_{\sigma_{1}}}\right) & \cdots & \pi_{1}\left(\mathbf{x}^{\alpha_{\sigma_{d}}}\right) \\
\vdots & \vdots & \ddots & \vdots \\
\pi_{d}\left(\mathbf{x}^{\alpha_{\sigma_{0}}}\right) & \pi_{d}\left(\mathbf{x}^{\alpha_{\sigma_{1}}}\right) & \cdots & \pi_{d}\left(\mathbf{x}^{\alpha_{\sigma_{d}}}\right)
\end{array}\right| \text {. }
$$

The above identity shows that if generic coefficients are assumed in the polynomial system, then the support of the Dixon polynomial depends entirely on the support of the polynomial system. To emphasize the dependence of $\Theta$ on $\mathcal{A}$, the above identity can also be written as $\theta_{\mathcal{A}}=\sum_{\sigma \in \mathcal{A}} \theta_{\sigma}$.

We define the support of the Dixon polynomial as:

$$
\Delta_{\mathcal{A}}=\left\{\alpha \mid \mathbf{x}^{\alpha} \in \theta\left(f_{0}, \ldots, f_{d}\right)\right\}
$$

where $\mathcal{A}=\left\langle\mathcal{A}_{0}, \ldots, \mathcal{A}_{d}\right\rangle, \mathcal{A}_{i}$ is the support of $f_{i}$. Let

$$
\bar{\Delta}_{\mathcal{A}}=\left\{\alpha \mid \overline{\mathbf{x}}^{\alpha} \in \theta\left(f_{0}, \ldots, f_{d}\right)\right\} .
$$

For the generic case, using the Cauchy-Binet formula,

$$
\Delta_{\mathcal{A}}=\bigcup_{\sigma \in \mathcal{A}} \Delta_{\sigma}
$$

because of genericity, $\theta_{\sigma}$ does not cancel any part of $\theta_{\tau}$ for any $\sigma, \tau \in \mathcal{A}$ and $\sigma \neq \tau$.

One of the properties of $\sigma(\mathbf{x})$, we will use, is that

$$
\Delta_{\sigma}=\left\{\alpha\left|\mathbf{x}^{\alpha} \in \theta_{\sigma}\right|_{\bar{x}_{i}=1}\right\},
$$

that is, substituting $\bar{x}_{i}=1$ for $i=1, \ldots, d$, does not change the support of the Dixon polynomial. This can be seen by noting that given a monomial in the expansion of the determinant of $\sigma(\mathbf{x})$ in terms of variables $x_{1}, \ldots, x_{d}$, its coefficient in terms of variables $\bar{x}_{1}, \ldots, \bar{x}_{d}$ can be uniquely identified. Hence, substituting $\bar{x}_{i}=1$ will not cancel any monomials as if there was cancellation, it should happen without substitution in the first place.

\section{Dixon Multiplier Matrix}

We define a Dixon multiplier matrix which is related to the Dixon matrix in the same way as Sylvester matrix is related to Bezout's; the first relationship generalizes the the second. This formulation also generalizes some of the earlier results which first appeared in [CK00b].

\subsection{Construction}

Let $m$ be a monomial in variables $\left\{x_{1}, x_{2}, \ldots, x_{d}\right\}$. For abbreviation, let

$$
\begin{aligned}
\theta & =\theta\left(f_{0}, f_{1}, \ldots, f_{d}\right), \quad \text { and also } \\
\theta_{i}(m) & =\theta\left(f_{0}, \ldots, f_{i-1}, m, f_{i+1}, \ldots, f_{d}\right) .
\end{aligned}
$$

Recall that in [CK00b], $\theta_{i}=\theta_{i}(1)=\theta\left(f_{0}, \ldots, f_{i-1}, 1, f_{i+1}, \ldots, f_{d}\right)$.

Theorem 3.1

$$
m \theta\left(f_{0}, \ldots, f_{d}\right)=\sum_{i=0}^{d} f_{i} \theta_{i}(m)
$$


In the case where $m=1$, the above identity was already used in [CK00b] as well [CM96] to show that the Dixon polynomial is in the ideal of the original polynomial system. For unmixed polynomial systems, using a general monomial $m$ enables us to build smaller Dixon multiplier matrices as there is a choice in selecting $m$. Note that it is crucial that $m$ does not vanish on any solution of the polynomial system $\mathcal{F}$.

In bilinear form $\theta_{i}(m)=\bar{X}_{i} \Theta_{i}(m) X_{i}$, where $\Theta_{i}(m)$ is the Dixon matrix of $\left\{f_{0}, \ldots, f_{i-1}, m, f_{i+1}, \ldots, f_{d}\right\}$. Expressing $\theta_{i}(m)$ in the term of $\Theta_{i}(m)$ matrix, we have $\theta_{i}(m) f_{i}=\left(\bar{X}_{i} \Theta_{i}(m) X_{i}\right) f_{i}=\left(\bar{X}_{i} \Theta_{i}(m)\right)\left(X_{i} f_{i}\right)$. Thus, we can construct a multiplier matrix by using monomial multipliers $X_{i}$ for $f_{i}$. Using the above notation, we can rewrite the formula for the Dixon matrix,

$$
\begin{aligned}
m \theta\left(f_{0}, \ldots, f_{d}\right) & =\bar{X} \Theta m X=\sum_{i=0}^{d} \theta_{i}(m) f_{i}=\sum_{i=0}^{d} \bar{X}_{i} \Theta_{i}(m) X_{i} f_{i} \\
& =\bar{Y}\left(\Theta_{0}(m): \cdots: \Theta_{d}(m)\right)\left(\begin{array}{c}
X_{0} f_{0} \\
X_{1} f_{1} \\
\vdots \\
X_{d} f_{d}
\end{array}\right) \\
& =\bar{Y}(T \times M) Y=\bar{Y} \Theta^{\prime} Y,
\end{aligned}
$$

where $\bar{Y}=\bigcup_{i=0}^{d} \bar{X}_{i}$ and $\Theta^{\prime}=T \times M$. Therefore,

$$
\bar{X} \Theta m X=\bar{Y} \Theta^{\prime} Y \text {. }
$$

Note that $m X \subseteq Y$, and $\bar{X} \subseteq \bar{Y}$; therefore, $\Theta$ and $\Theta^{\prime}$ are the same matrices except for $\Theta^{\prime}$ having some extra zero rows and columns.

\subsection{Maximal Minors}

It was proved in [KLS95] that under certain conditions, any maximal minor of the Dixon matrix is a projection operator (i.e., the nontrivial multiple of the resultant). More recently [BEM00] has established that any maximal minor of the Dixon matrix is a projection operator of a certain variety which is the projective closure of the associated affine set. These results immediately apply to the Dixon multiplier matrix, establishing that it is a resultant matrix.

Consider the following "specialization" map $\phi$ such that given a polynomial $f=\sum_{\alpha} c_{\alpha} \mathbf{x}^{\alpha}$,

$$
\phi(f)=\sum_{\alpha} \phi\left(c_{\alpha}\right) \mathbf{x}^{\alpha} \quad \text { and } \quad \phi\left(c_{\alpha}\right) \in \mathbb{C} \text {, so that } f \in \mathbb{C}[\mathbf{x}] .
$$

Theorem 3.2 ([KS96],[BEM00]) The determinant of a maximal minor of the Dixon matrix $\Theta$ of a polynomial system $\mathcal{F}$, is a projection operator, that is

$$
\operatorname{det}\left(\max _{\text {minor }}(\Theta)\right)=e R
$$

where $R$ is the resultant of the polynomial system $\mathcal{F}$, that is, $\phi(\mathcal{F}) \equiv 0$ has a solution if and only if $\phi(R)=0$.

The next theorem shows that the resultant is a factor in the projection operator computed from the Dixon multiplier matrix using maximal minor construction. Note that in the construction of a Dixon multiplier matrix, we must assume that for a map $\phi$ such that $\phi(\mathcal{F}) \equiv 0$ has a solution in the respective variety, $\phi(m) \neq 0$ for the monomial $m$ used to construct the Dixon multiplier matrix. This is the case when the common root is in is $\left(\mathbb{C}^{*}\right)^{d}$.

Theorem 3.3 The Dixon multiplier matrix is a resultant matrix, that is,

$$
\operatorname{det}\left(\max _{\text {minor }}(M)\right)=e R,
$$

where $R$ is the resultant of the polynomial system $\mathcal{F}$. 
The proof of this theorem is based on the fact that $\Theta=T \times M$ and that the Dixon Matrix $\Theta$ is the resultant matrix. $R$ divides determinant of maximal minor of $M$, and not $T$ since it is the condition for the existence of a solution of the polynomial system, and matrix $M$ represents that polynomial system. Another implication of this relationship of $\Theta$ and $M$ is that the gcd of all maximal minors of $T$ appears as a factor, among others, in any projection operator computed from the Dixon matrix.

\subsection{Multiplier Sets using the Dixon Method}

There is an interesting relationship between the multipliers used in the construction of a Dixon multiplier matrix and the monomials of the Dixon polynomial, which also label the columns of the Dixon matrix.

Proposition 3.1 Given the support $\mathcal{A}$ of a polynomial system $\mathcal{F}$, let

$$
\mathcal{A}(i, \alpha)=\left\langle\mathcal{A}_{0}, \ldots, \mathcal{A}_{i-1},\{\alpha\}, \mathcal{A}_{i+1}, \ldots, \mathcal{A}_{d}\right\rangle,
$$

where $\theta_{\mathcal{A}(i, \alpha)}=\theta_{i}\left(\mathbf{x}^{\alpha}\right)$. Then, for any $\alpha \in \mathbb{N}^{d}$,

$$
\Delta_{\mathcal{A}} \subseteq \bigcup_{i=0}^{d} \Delta_{\mathcal{A}(i, \alpha)} .
$$

Proof: Note that $\Delta_{\mathcal{A}(i, \alpha)}$ is the support of $\theta_{i}\left(\mathbf{x}^{\alpha}\right)$. Since $\mathbf{x}^{\alpha} \theta\left(f_{0}, \ldots, f_{d}\right)=\sum_{i=0}^{d} f_{i} \theta_{i}\left(\mathbf{x}^{\alpha}\right)$, we can conclude that

$$
\bar{\Delta}_{\mathcal{A}} \subseteq \bigcup_{i=0}^{d} \bar{\Delta}_{\mathcal{A}(i, \alpha)},
$$

since no $f_{i}$ has terms in variables $\bar{x}_{i}$. The Dixon polynomial depends on the variable order used in its construction. Let $\Delta_{\mathcal{A}}^{\left\langle x_{1}, x_{2}, \ldots, x_{d}\right\rangle}$ stand for the support of the Dixon polynomial constructed using the variable order $\left\langle x_{1}, x_{2}, \ldots, x_{d}\right\rangle$, i.e., $x_{1}$ is first replaced, followed by $x_{2}$ and so on. Therefore

$$
\bar{\Delta}_{\mathcal{A}}^{\left\langle x_{d}, \ldots, x_{1}\right\rangle} \subseteq \bigcup_{i=0}^{d} \bar{\Delta}_{\mathcal{A}(i, \alpha)}^{\left\langle x_{d}, \ldots, x_{1}\right\rangle} .
$$

However, $\bar{\Delta}_{\mathcal{A}}^{\left\langle x_{d}, \ldots, x_{1}\right\rangle}=\Delta_{\mathcal{A}}^{\left\langle x_{1}, \ldots, x_{d}\right\rangle}$, as can be seen from the definition 2.2 of $\theta$. The statement of the proposition then follows from the above three relations.

Proposition 3.2 Let $\alpha \in \mathcal{A}_{k}$ for all $k \in\{0, \ldots, d\}$; then, $\bigcup_{i=0}^{d} \Delta_{\mathcal{A}(i, \alpha)} \subseteq \Delta_{\mathcal{A}}$.

From Propositions 3.1 and 3.2, we have:

Theorem 3.4 Let $\alpha \in \mathcal{A}_{k}$ for all $k \in\{0, \ldots, d\}$, then

$$
\Delta_{\mathcal{A}}=\bigcup_{i=0}^{d} \Delta_{\mathcal{A}(i, \alpha)} .
$$

In particular, for an unmixed $\mathcal{A}, \Delta_{\mathcal{A}}=\Delta_{\mathcal{A}(i, \alpha)}$ for any $i$ and $\alpha \in \mathcal{A}_{i}$. The above property shows that the columns of the Dixon matrix are exactly the monomial multipliers of the Dixon multiplier matrix.

\subsection{Dixon-Invariant Support Points}

Definition 3.1 A point (degree exponent) $\alpha \in \mathbb{N}^{d}$ of a polynomial $f_{i}$ is called Dixon-invariant w.r.t. $\mathcal{A}_{i}$ for some $i \in\{0, \ldots, d\}$ of a polynomial system with support $\mathcal{A}=\left\langle\mathcal{A}_{0}, \ldots, \mathcal{A}_{d}\right\rangle$ if

$$
\Delta_{\left\langle\mathcal{A}_{0}, \ldots, \mathcal{A}_{i} \cup\{\alpha\}, \ldots, \mathcal{A}_{d}\right\rangle}=\Delta_{\left\langle\mathcal{A}_{0}, \ldots, \mathcal{A}_{d}\right\rangle},
$$

that is, the generic presence of the monomial corresponding to this point in the polynomial system does not influence the size of the associated Dixon matrix. Let $\widetilde{\mathcal{A}_{i}}$ denote the set of all Dixon-invariant points w.r.t. $\mathcal{A}_{i}$. 
In the unmixed case, a Dixon-invariant point is interior to the convex hull of the support, i.e., its Newton polytope, but the converse does not hold. Including a monomial corresponding to an arbitrary point interior w.r.t. a Newton polytope can, however, increase the size of the Dixon matrix.

In the following discussion, Dixon-invariant points will be shown to be among the best choice for a monomial $m$ in Dixon multiplier matrices; later they will also be related to the support of a polynomial system. In a later section, we give a geometric description of such points within a support and the associated Newton polytope.

\subsection{Choosing a Monomial for Constructing Multiplier Sets}

The choice of a monomial $m$ for constructing the Dixon multiplier matrix affects its size and structure. Our goal is to choose $m$ so as to produce the smallest possible resultant matrix for a given polynomial system, as then the extraneous factor appearing in a projection operator is minimized. It is proved below that choosing $m=\mathrm{x}^{\alpha}$ where $\alpha$ is Dixon-invariant produces the smallest Dixon multiplier matrices.

Theorem 3.5 For any $\alpha, \beta \in \bigcup_{i=0}^{d} \widetilde{\mathcal{A}}_{i}$,

$$
\left|M_{\alpha}\right|=\left|M_{\beta}\right|=\min _{\gamma \in \mathbb{N}^{d}}\left|M_{\gamma}\right|
$$

where $M_{\alpha}$ and $M_{\beta}$ are Dixon multiplier matrices constructed using monomial $m=\mathbf{x}^{\alpha}$ and $m=\mathbf{x}^{\beta}$ respectively.

The proof of the theorem is a direct consequence of Lemma 3.1 below.

Lemma 3.1 Let $k \in\{0,1, \ldots, d\}$, and $\beta \in \mathbb{N}^{d}$. If $\alpha \in \bigcap_{\substack{i \neq 0 \\ i \neq k}}^{d} \widetilde{\mathcal{A}}_{i}, \Delta_{\mathcal{A}(k, \alpha)} \subseteq \Delta_{\mathcal{A}(k, \beta)}$.

Proof: $\operatorname{card}_{\mathcal{A}}(\alpha)=d$. Using Lemma 3.2 proved below, it follows that $\Delta_{\mathcal{A}(k, \beta)}=\Delta_{\mathcal{A}(k,\{\beta\} \cup\{\alpha\})}$; since $\Delta_{\mathcal{A}(k, \alpha)} \subseteq \Delta_{\mathcal{A}(k,\{\beta\} \cup\{\alpha\})}$, the statement of the lemma follows.

Given $\alpha \in \mathbb{N}^{d}$, and a polynomial system support $\mathcal{A}=\left\langle\mathcal{A}_{0}, \ldots, \mathcal{A}_{d}\right\rangle$, let card ${ }_{\mathcal{A}}(\alpha)$ denote the number of indices $i$ such that each $\alpha \in \mathcal{A}_{i}$.

Lemma 3.2 Given a polynomial system with support $\mathcal{A}=\left\langle\mathcal{A}_{0}, \ldots, \mathcal{A}_{d}\right\rangle$, let $\mathcal{P}=\left\langle\mathcal{P}_{0}, \ldots, \mathcal{P}_{d}\right\rangle$ be an unmixed polynomial system support such that $\mathcal{P}_{i}=\mathcal{A}_{0} \cup \cdots \cup \mathcal{A}_{d}$. If $\operatorname{card}_{\mathcal{A}}(\alpha) \geq d$, then $\Delta_{\mathcal{A}}=\Delta_{\mathcal{P}}$.

Proof: Clearly $\mathcal{A} \subseteq \mathcal{P}$ as for any $\sigma \in \mathcal{A}, \sigma \in \mathcal{P}$. To show that $\Delta_{\mathcal{A}} \supseteq \Delta_{\mathcal{P}}$, note that for any $\sigma=\left\langle\sigma_{0}, \ldots, \sigma_{d}\right\rangle$ the order of $\sigma$ 's does not change $\Delta_{\sigma}$. Since $\operatorname{card}_{\mathcal{A}}(\alpha) \geq d$, it is always possible for any $\sigma \in \mathcal{P}$ to rearrange $\sigma$ so that $\sigma_{i} \in \mathcal{A}_{i}$ and hence $\sigma \in \mathcal{A}$. Therefore $\Delta_{\mathcal{A}} \supseteq \Delta_{\mathcal{P}}$.

Lemma 3.1 states that for any choice of $m=\mathbf{x}^{\beta}$, the set of multipliers for $f_{k}$ must include all the multipliers in case $m$ is chosen to be $\mathbf{x}^{\alpha}$. If $\bigcap_{i=0}^{d} \widetilde{\mathcal{A}}_{i}$ is non-empty, then any choice of $\alpha$ in $\bigcap_{i=0}^{d} \widetilde{\mathcal{A}}_{i}$ will yield matrices of smallest size.

On the other hand, in the unlikely case when $\bigcap_{i=0}^{d} \widetilde{\mathcal{A}}_{i}=\oslash$, supports $\mathcal{A}_{i}$ can be translated so that the intersection is not empty. Even in the case of non-empty intersection of supports, this heuristic of translating supports might be beneficial in minimizing the size of Dixon multiplier matrix; an exact analysis of this needs further study.

\subsection{Size of Dixon Multiplier Matrix}

Theorem 3.4 implies that in unmixed cases, for $\mathcal{A}=\left\langle\mathcal{A}_{0}, \ldots, \mathcal{A}_{d}\right\rangle$ where $\mathcal{A}_{i}=\mathcal{A}_{j}$, if $\alpha \in \widetilde{\mathcal{A}}_{i}$ then

$$
(d+1) \operatorname{size}(\Theta)=\operatorname{size}\left(M_{\alpha}\right),
$$

that is, the size of the Dixon multiplier matrix is exactly $d+1$ times bigger. We also have:

Theorem 3.6 Given a generic, unmixed polynomial system $\mathcal{F}$ and a monomial $m$ in $\mathcal{F}$ (or $m$ being Dixon invariant in $\mathcal{F})$, then if the Dixon matrix is exact, then the Dixon multiplier matrix built with $\theta_{i}(m)$ is also exact. 
In mixed cases the ratio between sizes of two matrices is at most $d+1$, therefore, Dixon multiplier matrices are as good as Dixon matrices in unmixed cases, in terms of extraneous factors and usually better in mixed cases.

\section{Support of the Dixon Polynomial}

The support of the Dixon polynomial of a generic polynomial system with support $\mathcal{A}$ admits a geometric description and is, therefore, practically more advantageous to compute than the direct expansion of the Dixon polynomial.

\subsection{Support Interior}

Definition 4.1 Given $k \in \mathbb{Z}_{2}^{d}$ and $p, q \in \mathbb{N}^{d}$, define

$$
p \underset{k}{<} q \quad \text { if } \quad \begin{cases}p_{j}<q_{j} & \text { if } k_{j}=1, \\ p_{j} \geq q_{j} & \text { if } k_{j}=0 .\end{cases}
$$

Note that from above, $p_{j}$ is strictly smaller that $q_{j}$, but from below, $p_{j}$ is equal or greater that $q_{j}$. If equality is also permitted from above, it will be denoted by $p \frac{<}{k} q$. For fixed $k$, this relation is transitive, but it is not a total order.

Given a point $p$ and any set $\mathcal{P} \subset \mathbb{N}^{d}$, denote by

$$
p \triangleleft \mathcal{P} \Longleftrightarrow \forall k \in \mathbb{Z}_{2}^{d}, \exists q \in \mathcal{P} \text { s.t. } p_{k}^{<} q,
$$

and similarly by $p \unlhd \mathcal{P}$, if for in the above condition $p \stackrel{\leq}{k} q$. Let

$$
\widehat{\mathcal{P}}=\{p \mid p \unlhd \mathcal{P}\},
$$

denote thesupport $\mathcal{P}$ closed with its support interior points.

Given the support of a polynomial, its convex hull is the Newton polytope associated with the polynomial. The BKK bound on the number of roots discussed above tells us that the degree of the resultant is determined by the mixed volume of the Newton polytopes of the polynomials. In this sense, points strictly interior in the Newton polytope, i.e., the convex hull, do not change the degree of the resultant. It will be ideal to have a method for constructing resultant matrices such that the size of the resultant matrix is determined by the Newton polytope of the polynomials, but in practice, for all methods, points interior to the Newton polytope do affect the size of the resultant matrix; including additional points interior to the Newton polytope usually result in bigger resultant matrices with their determinants having additional extraneous factors besides the resultants.

We analyze the role of points interior to a Newton polytope vis a vis the size of Dixon-based resultant matrices. Since the size of the Dixon matrix as well as the Dixon multiplier matrix are determined by the support of the Dixon polynomial, we identify points within a Newton polytope such that whenever monomials corresponding to these points are introduced generically in a polynomial system, the structure of the associated Dixon polynomial and hence, the size of the Dixon matrix remains invariant.

Clearly $\widehat{\mathcal{P}} \subseteq \operatorname{Conv}(\mathcal{P}) \cap \mathbb{Z}^{d}$, i.e., support interior is a subset of the Newton polytope. Below we show that support interior points do not affect the size and the structure of the support of the Dixon polynomial, and hence, the degree of the projection operator computed using the Dixon formulation. This is similar to the mixed volume, where points inside the convex hull do not change the number of roots polynomial system has, and hence the resultant degree. A proof of the following theorem is given in [CK02c].

Theorem 4.1 Given an unmixed polynomial system $\mathcal{A}=\left\langle\mathcal{A}_{0}, \ldots, \mathcal{A}_{d}\right\rangle$,

$$
\widehat{\mathcal{A}}=\widetilde{\mathcal{A}},
$$

i.e., a support interior point is Dixon-invariant and a Dixon-invariant point is support interior. 


\section{Unmixed Polynomial Systems, Exact Cases for Dixon Method}

Consider an unmixed polynomial system $\langle\mathcal{A}, \mathcal{A}, \ldots, \mathcal{A}\rangle$. Assume that $\mathcal{A}$ is situated at the origin, in the first octant, that is, $\exists \alpha \in \mathcal{A}$ s.t. $\forall i \in\{1, \ldots, d\}, \alpha_{i}=0$. We will call such a support to be cornered. Given a cornered support $\mathcal{A}=\left\{\alpha_{1}, \ldots, \alpha_{n}\right\}$, let $b_{i}=\max _{j=1}^{n} \alpha_{j, i}$.

Definition 5.1 Given a support $\mathcal{A}$, define its box to be

$$
\mathcal{B}=\left\{p=\left(p_{1}, \ldots, p_{d}\right) \mid 0 \leq p_{i} \leq b_{i}\right\} .
$$

An unmixed polynomial system with the support $\mathcal{B}$ is called $n$-degree. Note that $\hat{\mathcal{B}}=\mathcal{B}$.

Proposition 5.1 The support of the Dixon polynomial of an n-degree generic polynomial system is

$$
\Delta_{\mathcal{B}}=\left\{p=\left(p_{1}, \ldots, p_{d}\right) \mid 0 \leq p_{i}<i b_{i}\right\} .
$$

Hence, $\left|\Delta_{\mathcal{B}}\right|=d ! \operatorname{Vol}(\mathcal{B})=d ! \prod_{i=1}^{d} b_{i}$. The above was already established in [KS96] and [Sax97]. That is, Dixon-based matrices are exact for polynomial systems with $n$-degree support. Recently, [ZG00] has shown that exact multiplier matrices can be constructed in the bivariate case for polynomial systems whose support is bi-degree with corners removed. In [CK02a], we have generalized this result to show that Dixon matrices can be used to compute exact resultants for polynomial systems whose support is bi-degree with arbitrary corners removed. We generalize these ideas to the multivariate case; the results do not as yet provide a complete analysis for the multivariate case, but are still of practical and theoretical significance.

\subsection{Support Projections \& Variable Order}

The support of the Dixon polynomial is in essence the Minkowski sum of the projections of the supports of polynomials in the system. Depending on a variable order used in the construction (definition 2.2), different Dixon polynomials can be obtained, and more importantly, the size of the polynomials might not be the same. Smaller Dixon polynomial will result in smaller Dixon as well as Dixon multiplier matrix and hence, smaller extraneous factor in a projection operator.

\subsubsection{Projections of Supports}

In general, for any set $\mathcal{Q} \subset \mathbb{N}^{d}$, let $\mathcal{Q}_{1 \ldots i}$ stand for the $i^{t h}$ projection of $\mathcal{Q}$, i.e.,

$$
\begin{aligned}
& \mathcal{Q}_{1 \ldots i}=\left\{\left(a_{1}, \ldots, a_{i}, 0, \ldots, 0\right) \mid a \in \mathcal{Q}\right\} \\
& \mathcal{Q}_{1 \ldots i}^{*}=\left\{\left(a_{1}, \ldots, a_{i}, *, \ldots, *\right) \mid a \in \mathcal{Q}\right\},
\end{aligned}
$$

where $\left(a_{1}, \ldots, a_{i}, *, \ldots, *\right)$ is a set of points whose last $d-i$ coordinates are arbitrary.

Since we know the support of the Dixon polynomial for a generic polynomial system with a box support $\mathcal{B}$, for a generic polynomial system with support $\mathcal{A}$, we relate the difference between the support $\Delta_{\mathcal{B}}$ of the Dixon polynomial of the system with the box support $\mathcal{B}$ to the support $\Delta_{\mathcal{A}}$ of the Dixon polynomial of the system with the support $\mathcal{A}$ to the difference between the box support $\mathcal{B}$ and the support $\mathcal{A}$. Consider the complement of $\mathcal{A}$ in $\mathcal{B}$ for every projection ( - is the set difference operator): $\mathcal{C}_{1 \ldots i}=\mathcal{B}_{1 \ldots i}-\widehat{\mathcal{A}_{1 \ldots i}}$ and sub-complement $S_{1 \ldots i}=\mathcal{C}_{1 \ldots i}-\mathcal{C}_{1 \ldots i-1}^{*}$. In all cases, $\left|S_{1 \ldots 1}\right|=0$, as $\mathcal{B}_{1 \ldots 1}=\widehat{\mathcal{A}_{1 \ldots 1}}$. An important property of sub-complements is that they are disjoint for any projection, that is, $S_{1 \ldots i}^{*} \cap S_{1 \ldots j}^{*}=\oslash$ for $i \neq j$, which comes from the fact that $\mathcal{C}_{1 \ldots j}^{*} \cap \mathcal{B} \subseteq \mathcal{C}_{1 \ldots i}$ for $j<i$. Also, note that $\mathcal{A}=\mathcal{B}-\bigcup_{i=1}^{d} S_{1 \ldots i}^{*}$.

Given $S_{1 \ldots i}$, it can be split into $2^{i}$ pieces as follows. For every $k \in \mathbb{Z}_{2}^{i}$, define a set $S_{1 \ldots i}^{k}$, where a point $p \in S_{1 \ldots i}$, belongs to $S_{1 \ldots i}^{k}$ if there does not exist $\alpha \in \mathcal{A}$ such that $p \underset{k}{\stackrel{\leq}{\alpha}} \alpha$.

$$
S_{1 \ldots i}^{k}=\left\{p \mid p \in S_{1 \ldots i} \text { and } \nexists \alpha \in \mathcal{A} \text { s.t. } p_{\bar{k}}^{\leq} \alpha\right\} .
$$


The union of all $S_{1 \ldots i}^{k}$ is the entire set $S_{1 \ldots i}$, i.e., $S_{1 \ldots i}=\bigcup_{k \in \mathbb{Z}_{2}^{i}} S_{1 \ldots i}^{k}$ but $S_{1 \ldots i}^{k}$ 's are not necessarily disjoint for different $k$ 's.

Since for $\mathcal{A}$, the support complement $S_{1 \ldots i}^{*}$ is a part which is "missing" from $\mathcal{B}$, there is a corresponding part for $\Delta_{\mathcal{A}}$ which is "missing" from $\Delta_{\mathcal{B}}$. This is similar to the analysis done for the bivariate case in [CK02a].

Definition 5.2 Let $k \in \mathbb{Z}_{2}^{d}$, and $T_{1 \ldots i}^{k}=r^{k}+S_{1 \ldots i}^{k}$ and set $T_{1 \ldots i}=\bigcup_{k \in \mathbb{Z}_{2}^{d}} T_{1 \ldots i}^{k}$, where for $r^{k}=$ $\left(r_{1}^{k}, \ldots, r_{d}^{k}\right) \in \mathbb{N}^{d}$,

$$
r_{j}^{k}=\left\{\begin{array}{cl}
(j-1) b_{j}-1 & \text { if } k_{j}=1 \\
0 & \text { if } k_{j}=0
\end{array}\right.
$$

In addition to $\Delta_{\mathcal{A}} \subseteq \Delta_{\mathcal{B}}$, we have:

Theorem 5.1 $\Delta_{\mathcal{A}} \subseteq \Delta_{\mathcal{B}}-\bigcup_{i=1}^{d} T_{1 \ldots i}^{*}$.

To estimate an upper bound of the support of the Dixon polynomial, a number of properties of $T_{1 \ldots i}$ are needed. The proofs are given in [CK02b].

Proposition 5.2 For $i, j \in\{0, \ldots, d\}$,

(i) $T_{1 \ldots i}^{k} \cap T_{1 \ldots i}^{l}=\oslash \quad$ for $\quad k, l \in \mathbb{Z}_{2}^{i} \quad$ and $\quad k \neq l$,

(ii) $T_{1 \ldots i}^{*} \cap T_{1 \ldots j}^{*}=\oslash$ for $i \neq j$,

(iii) $\left|T_{1 \ldots i}\right|=\sum_{k \in \mathbb{Z}_{2}^{i}}\left|S_{1 \ldots i}^{k}\right|$.

Using these properties along with $\left|T_{1 \ldots i}^{*} \cap \Delta_{\mathcal{B}}\right|=\frac{d !}{i !}\left|T_{1 \ldots i}\right| \prod_{j=i+1}^{d} b_{j}$, we get the following inequality:

$$
\left|\Delta_{\mathcal{A}}\right| \leq d ! \prod_{i=1}^{d} b_{i}-\sum_{i=1}^{d}\left(\frac{d !}{i !}\left|T_{1 \ldots i}\right| \prod_{j=i+1}^{d} b_{j}\right) \text {. }
$$

\subsection{Exact Cases}

For supports $\mathcal{A}$ for which the inequality (1) becomes equality, the matrices constructed using the Dixon formulation are exact. In other words,

$$
\left|\Delta_{\mathcal{A}}\right|=d ! \prod_{i=1}^{d} b_{i}-\sum_{i=1}^{d}\left(\frac{d !}{i !}\left|T_{1 \ldots i}\right| \prod_{j=i+1}^{d} b_{j}\right) \Longrightarrow\left|\Delta_{\mathcal{A}}\right|=d ! \operatorname{Vol}(\mathcal{A})
$$

The above implication defines a broad class of supports for which the Dixon formulation produces exact resultants. It generalizes most known unmixed exact cases, including $n$-degree systems [KS96], bivariate corner-cut supports [ZG00, Chi01], as well as their generalizations discussed in [CK02a].

For example, for an $n$-degree system, each set $S_{1 \ldots i}=\oslash$; hence, $\left|T_{1 \ldots i}\right|=0$ and $\left|\Delta_{\mathcal{A}}\right|=\left|\Delta_{\mathcal{B}}\right|$ and therefore $\left|\Delta_{\mathcal{A}}\right|=d ! \prod_{i=1}^{d} b_{i}=d ! \operatorname{Vol}(\mathcal{A})$.

Below, a generalization of a bivariate corner-cut support for which the Dixon based methods compute the exact resultant, to an arbitrary dimension is discussed.

Given $\mathcal{A}$, define $\mathcal{A}_{-i}$ to be the projection of $\mathcal{A}$ on the $i^{\text {th }}$ coordinate, i.e.,

$$
\mathcal{A}_{-i}=\left\{\left(a_{1}, \ldots, a_{i-1}, a_{i+1}, \ldots, a_{d}\right) \mid\right.
$$

$$
\left.\left(a_{1}, \ldots, a_{i-1}, a_{i}, a_{i+1}, \ldots, a_{d}\right) \in \mathcal{A}\right\}
$$


1. Corner-cut: $\mathcal{A}$ is called corner-cut if and only if for all $i \in\{1, \ldots, d\}$,

$$
\begin{aligned}
\widehat{\mathcal{A}}_{-i}= & \mathcal{B}_{-i}, \text { and } \\
S_{1 \ldots d}^{k} & \text { is a } d \text {-dimensional rectangle } \\
& \text { for each } k \in \mathbb{Z}_{2}^{d} .
\end{aligned}
$$

2. Almost Corner-cut: $\mathcal{A}$ is called almost corner-cut if there is some $j \in\{1, \ldots, d\}$ such that for each $i \in\{1, \ldots, d\}, i \neq j$ and

$$
\begin{aligned}
\widehat{\mathcal{A}_{-i}}= & \mathcal{B}_{-i}, \\
S_{1 \ldots d}^{k} & \text { is a } d \text {-dimensional rectangle } \\
& \text { for all } k \in \mathbb{Z}_{2}^{d},
\end{aligned}
$$

and for each $k^{\prime} \in \mathbb{Z}_{2}^{d-1}, S_{-j}^{k^{\prime}}$ is a $d-1$ dimensional rectangle, where $S_{-j}^{k^{\prime}}$ is defined as $S_{1 \ldots(j-1)(j+1) \ldots d}^{k^{\prime}}$.

Theorem 5.2 ( $d$-dimensional Corner-Cut) Given an unmixed generic polynomial system with a corner-cut support $\mathcal{A}$, the Dixon Matrix is exact for any variable order used to construct it.

The notion of an almost corner-cut support is slightly more general than a corner-cut support; the $j^{\text {th }}$ coordinate for which $\widehat{\mathcal{A}_{-j}} \neq \mathcal{B}_{-j}$, corresponds to considering the variable $x_{j}$ last, in the variable order used to construct the Dixon polynomial.

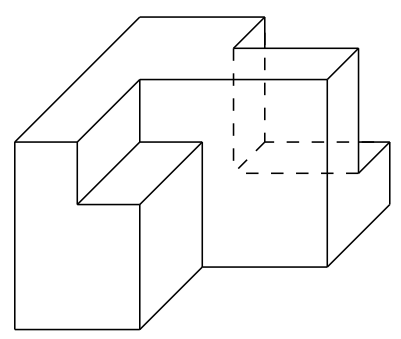

The example in the above figure is that of an almost corner-cut support; it is not corner-cut. Assuming that the vertical axis is the $z$ axis, it can be seen that $\widehat{\mathcal{A}_{-z}} \neq \mathcal{B}_{-z}$, but $S_{-z}^{k^{\prime}}$ is a rectangle. For other coordinates $\widehat{\mathcal{A}_{-x}}=\mathcal{B}_{-x}$ and $\widehat{\mathcal{A}_{-y}}=\mathcal{B}_{-y}$.

Theorem 5.3 (Almost Corner-Cut) Given an unmixed generic polynomial system with an almost corner-cut support $\mathcal{A}$, the Dixon Matrix is exact for any variable order used to construct it except that the last variable in the variable order is $x_{j}$.

Due to space limitations, proofs of these theorems could not be included in the paper. An interested reader is referred to the technical report [CK02b] for detailed proofs.

We have thus settled an open problem raised in [ZG00] of generalizing corner cut supports to multidimensional cases. Corner-cut support can be generalized in many different ways; it is shown above that the Dixon formulation is exact for corner-cut and almost corner-cut supports as defined above. There are families of generic unmixed polynomial systems whose support is neither corner-cut nor almost corner-cut, yet the Dixon formulation still produces exact resultants. A notable such family is that of multigraded systems introduced in [MS87] and analyzed for Dixon construction in [CK00a].

\subsection{A Heuristic for Minimizing the Degree of the Extraneous Factor}

Based on the above inequality (1), the following greedy heuristic for picking the best variable order to construct the Dixon Matrix is proposed. The main objective is to maximize $\left|T_{1 \ldots i}\right| \prod_{j=i+1}^{d} b_{j}$ for the smallest $i$. Since for $i=1,\left|T_{1 \ldots i}\right|=0$, one need to choose first two coordinates so that $\left|T_{1 \ldots 2}\right| \prod_{j=3}^{d} b_{j}$ is maximized. Trying all combinations of two variables, first two variables can be fixed in $O\left(d^{2}\right)$ times the cost of computing $\left|T_{1 \ldots 2}\right| \prod_{j=3}^{d} b_{j}$. (One can estimate the size of $T_{1 \ldots i}$ in linear time in terms of the number of points in $\mathcal{A}$ if intermediate values are computed and stored using appropriate data structures, which can be done at the cost of $O\left(2^{d} n\right)$ as shown in [CK02b]). Third variable can be fixed only after $d-2$ calculations of $\left|T_{1 \ldots 3}\right| \prod_{j=4}^{d} b_{j}$, fourth after $d-3$ and so on; this can be done at a cost not exceeding $O\left(d^{2} n\right)$. So the best variable order can be established in $O\left(n^{d}\right)$ (without increasing the complexity of 
computing the Dixon matrix) where the brute force approach is of $O\left(d ! n^{d}\right)$ as each evaluation of the Dixon polynomial is $O\left(n^{d}\right)$.

This heuristic reduces the right hand side of the above inequality to a minimum. For cases where the inequality becomes equality, this procedure will establish an optimal variable order. It has a limitation, however; in the unlikely case where most of the $\left|T_{1 \ldots i}\right|$ are the same for different combinations of coordinates, the above heuristic may not lead to a best variable order. Nevertheless, without incurring additional cost of computing, it is possible to find ways to minimize the size of Dixon matrix and hence the degree of the projection operator.

\subsection{Summary}

Conditions on supports have been identified for which the Dixon based resultant formulations compute exact resultants of polynomial systems in the generic unmixed case. These conditions are an attempt to generalize the concept of corner-cut support introduced in [Chi01, ZG00, CK02a] for the bivariate case. Whereas in the bivariate case, corner-cut supports are a necessary and sufficient condition on bivariate generic unmixed polynomial systems for which the Dixon-based formulations generate exact resultants, such a characterization for general multivariate generic unmixed polynomial systems is not known and is thus worth investigating.

\section{References}

[BEM00] Laurent Buse, Mohamed Elkadi, and Bernar Mourrain. Generalized resultants over unirational algebraic varieties. J. Symbolic Computation, 29:515-526, 2000.

[CE00] J.F. Canny and I.Z. Emiris. A subdivision based algorithm for the sparse resultant. J. ACM, 2000 .

[Chi01] E.-W. Chionh. Rectangular corner cutting and dixon $\mathcal{A}$-resultants. Journal of Symbolic Computation, 31:651-663, 2001.

[CK00a] A. D. Chthcerba and D. Kapur. Conditions for exact resultants using the Dixon formulation. ISSAC00, pages 62-70, 2000 .

[CK00b] A. D. Chthcerba and D. Kapur. Extracting sparse resultant matrices from the Dixon resultant formultation. Proc. of 'th Rhine Workshop (RCWA'00), pages 167-182, 2000.

[CK02a] A. D. Chthcerba and D. Kapur. A complete analysis of resultants and extraeneous factors for unmixed bivariate polynomial systems using the Dixon formulation. In Proc. of 8th Rhine Workshop (RCWA'02), pages 137-165, Mannheim, Germany, 2002.

[CK02b] A. D. Chthcerba and D. Kapur. Exact Resultants for corner-cut unmixed multivariate polynomial systems using the Dixon formulation. Technical report, Computer Science Dept., Univ. of New Mexico, Albuquerque, New Mexico, USA, 2002.

[CK02c] A. D. Chthcerba and D. Kapur. On Relationship between the Dixon-based Resultant Construction and the Supports of Polynomial Systems. Technical report, Computer Science Dept., Univ. of New Mexico, Albuquerque, New Mexico, USA, 2002.

[CLO98] D. Cox, J. Little, and D. O'Shea. Using Algebraic Geometry. Springer-Verlag, New York, first edition, 1998.

[CM96] J.P. Cardinal and B. Mourrain. Algebraic approach of residues and applications. In J. Renegar, M. Shub, and S. Smale, editors, AMS-SIAM Summer Seminar on Math. of Numerical Analysis, Lectures in Applied Mathematics, volume 32, pages 189-210. Am. Math. Soc. Press, 1996. 
[Dix08] A.L. Dixon. The eliminant of three quantics in two independent variables. Proc. London Mathematical Society, 6:468-478, 1908.

[EM99] Ioannis Z. Emiris and Bernard Mourrain. Matrices in elimination theory. Journal of Symbolic Computation, 28(1-2):3-43, 1999.

[GKZ94] I.M. Gelfand, M.M. Kapranov, and A.V. Zelevinsky. Discriminants, Resultants and Multidimensional Determinants. Birkhauser, Boston, first edition, 1994.

[KLS95] D. Kapur, Y.N. Lakshman, and T. Saxena. Computing invariants using elimination methods. In IEEE Symp. On Comp. Vision, pages 97-102, 1995.

[KS95] D. Kapur and T. Saxena. Comparison of various multivariate resultants. In ACM ISSAC 95, Montreal, Canada, July 1995.

[KS96] D. Kapur and T. Saxena. Sparsity considerations in the Dixon resultant formulation. In Proc. ACM Symposium on Theory of Computing, Philadelphia, May 1996.

[KS97] D. Kapur and T. Saxena. Extraneous factors in the Dixon resultant formulation. In ISSAC, pages 141-147, Maui, Hawaii, USA, 1997.

[KSY94] D. Kapur, T. Saxena, and L. Yang. Algebraic and geometric reasoning using the Dixon resultants. In ACM ISSAC 94, pages 99-107, Oxford, England, July 1994.

[Mac16] F.S. Macaulay. The algebric theory of modular systems. Cambridge Tracts in Math. and Math. Phys., 19, 1916.

[MS87] A.P. Morgan and A.J. Sommese. A homotopy for solving general polynomial systems that respects m-homogeneous structures. Appl. Math. Comput., 24:101-113, 1987.

[PS93] P.Pedersen and B. Sturmfels. Product formulas for resultants and chow forms. Math. Zeitschrift, 214:377-396, 1993.

[Sax97] T. Saxena. Efficient variable elimination using resultants. PhD thesis, Department of Computer Science, State Univeristy of New York, Albany, NY, 1997.

[Stu94] B. Sturmfels. On the newton polytope of the resultant. Journal of Algebraic Combinatorics, 3:207-236, 1994.

[Syl53] J.J Sylvester. On a theory of syzygetic relations of two rational integral functions, comprising an application to the theory of sturm's functions, and that of the greatest algebraic common measure. Philosophical Trans., 143:407-548, 1853.

[ZG00] Ming Zhang and Ron Goldman. Rectangular corner cutting and sylvester $\mathcal{A}$-resultants. In Carlo Traverso, editor, Proc. of the ISSAC, pages 301-308, St. Andrews, Scotland, 2000. ACM Press. 